\title{
Antarctic Cosmic Ray Astronomy
}

\author{
Marc Duldig \\ Australian Antarctic Division, Channel Highway, Kingston, Tasmania, \\ 7050, Australia
}

\begin{abstract}
Cosmic ray observations related to Antarctica commenced in the austral summer of 1947-48 from sub-Antarctic Heard and Macquarie Islands and from the HMAS Wyatt Earp. Muon telescope observations from Mawson station, Antarctica, followed from 1955. The International Geophysical Year was the impetus for the installation of a number of neutron monitors around Antarctica, observing the lowest energy cosmic rays accessible by ground based instruments. In 1971 a new observatory was built at Mawson including the only underground muon telescope system at polar latitudes in either hemisphere. Over more than half a century, cosmic ray astronomy has been undertaken from Antarctica and its surrounding regions and these observations have been critical to our growing understanding of the heliosphere.
\end{abstract}

\section{Introduction}

Cosmic rays are fully ionized relativistic particles arriving outside the earth from beyond the solar system. On rare occasions the sun can be a source of cosmic rays. At the lowest energies $(1-50 \mathrm{GeV})$ neutron monitors are employed to detect the radiation but these instruments have no directional capability. The viewing cone is defined by the particle trajectories through the geomagnetic field that can reach the atmosphere above the monitor. At higher energies $(\sim 10-1000 \mathrm{GeV})$ highly penetrating muons are produced in the atmospheric interaction and these may be detected by standard charged particle detectors arranged in multi-tray coincidence systems above or below ground. These muon telescopes effectively record the arrival direction of the initial cosmic ray above the atmosphere. For a discussion of the general properties of cosmic rays and their detection see Duldig (1994). The deflections experienced by cosmic rays traversing the heliomagnetic field can be used to tell us about the field's structures and its interactions with the local interstellar medium. The Antarctic platform provides a unique opportunity to study cosmic rays of low energy due to proximity to the magnetic pole and arrival directions not accessible from other places on earth.

\section{Observations and Results}

In the late 1940's Geoff Fenton began developing Geiger counters. He investigated the mid-latitude E-W effect from Hobart confirming the southern hemisphere showed the same effect as the north. Geiger counter telescopes and ionisation chambers were operated on the ship HMAS Wyatt Earp in the southern ocean and on the sub-Antarctic Heard and Macquarie Islands by groups 
from Melbourne and Tasmania Universities. Around 1950 Melbourne University withdrew from the cosmic ray observations in the sub-Antarctic. Muon telescopes were installed at Mawson in 1955, one year after the station opened and neutron monitors followed at several Antarctic stations in time for the IGY.

A large ground level enhancement (GLE) was observed in May 1960 with the global neutron monitor network. This resulted from acceleration of protons to cosmic ray energies associated with a flare on the Sun. The flare site was magnetically well connected to the earth and the Mawson observation of the response proved the existence of the Parker spiral field long before the in-situ measurement of the field by spacecraft (McCracken 1962). The discovery of the co-rotational anisotropy and its spectrum relied heavily on global observations and the Antarctic observations were critical to understanding the process.

In 1971 a new observatory was constructed at Mawson that includes the only underground muon observatory at polar latitudes. The underground telescopes were instrumental in the discovery of isotropic intensity waves by Jacklyn, Duldig \& Pomerantz (1987). In a landmark study (Bieber \& Chen 1991; Chen \& Bieber 1993) used polar neutron monitors to derive cosmic ray gradients and mean free paths in the heliosphere. This work was extended by Hall, Duldig \& Humble (1997) to higher energies by incorporating the muon telescopes' observations from Mawson and other sites. Nagashima, Fujimoto \& Jacklyn (1998) challenged conventional wisdom with a new interpretation of the sidereal anisotropy response observed. Further work employing Mawson and other muon telescope observations by Hall et al (1999) elucidated the structure more precisely.

\section{Summary}

The Antarctic region has been critical to understanding the cosmic ray flux at earth. Over half a century of instrumental development and observations have led to a deepening understanding of the heliosphere. Now cosmic ray measurements are providing promising input into space weather prediction. The future of Antarctic cosmic ray astronomy seems to be as bright as ever.

\section{References}

Bieber, J. W., \& Chen, J. 1991, ApJ, 372, 301

Chen, J., \& Bieber, J. W. 1993, ApJ, 405, 375

Duldig, M. 1994, PASA, 11, 114

Hall, D. L., Duldig, M. L., \& Humble, J. E. 1997, ApJ, 482, 1038

Hall, D. L., Munakata, K., Yasue, S., Mori, S., Kato, C., Koyama, M., Akahane, S., Fujii, Z., Fujimoto, K., Humble, J. E., Fenton A. G., Fenton, K. B., \& Duldig, M. L., 1999, JGR, 104, 6737

Jacklyn, R. M., Duldig, M. L., \& Pomerantz, M. A., 1987, JGR, 92, 8511.

McCracken, K. G., 1962, JGR, 67, 447

Nagashima, K., Fujimoto, K., \& Jacklyn, R. M. 1998, JGR, 103, 17429 